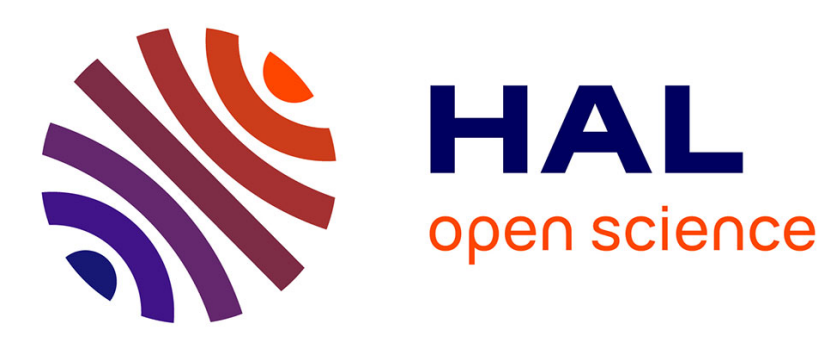

\title{
Biomimetic-Based Output Feedback for Attitude Stabilization of a Flapping-wing Micro Aerial Vehicle
}

Hala Rifai, Fermi Guerrero-Castellanos, Nicolas Marchand, Guylaine Poulin

\section{To cite this version:}

Hala Rifai, Fermi Guerrero-Castellanos, Nicolas Marchand, Guylaine Poulin. Biomimetic-Based Output Feedback for Attitude Stabilization of a Flapping-wing Micro Aerial Vehicle. Robotica, 2013, 31 (6), pp.955-968. 10.1017/S0263574713000209 . hal-00728410

\section{HAL Id: hal-00728410 https://hal.science/hal-00728410}

Submitted on 1 Apr 2015

HAL is a multi-disciplinary open access archive for the deposit and dissemination of scientific research documents, whether they are published or not. The documents may come from teaching and research institutions in France or abroad, or from public or private research centers.
L'archive ouverte pluridisciplinaire HAL, est destinée au dépôt et à la diffusion de documents scientifiques de niveau recherche, publiés ou non, émanant des établissements d'enseignement et de recherche français ou étrangers, des laboratoires publics ou privés. 


\title{
Biomimetic-Based Output Feedback for Attitude Stabilization of a Flapping-wing Micro Aerial Vehicle $\dagger$
}

\author{
H. Rifaï ${ }^{1}$, J.-F. Guerrero-Castellanos ${ }^{2}$, N. Marchand ${ }^{3}$, G. \\ Poulin-Vittrant ${ }^{4}$ \\ ${ }^{1}$ LISSI, 120-122 rue Paul Armangot, 94400 Vitry-Sur-Seine, FRANCE \\ ${ }^{2}$ Autonomous University of Puebla (BUAP), Faculty of Electronics, Puebla, MEXICO \\ ${ }^{3}$ GIPSA-lab - Control Systems department - ENSE3/CNRS, Saint Martin d'Hères, FRANCE \\ ${ }^{4}$ GREMAN, UMR 7347 CNRS - University François Rabelais de Tours, Site de Blois, Rue de \\ la Chocolaterie, 41000 Blois, FRANCE \\ emails: hala.rifai@u-pec.fr, fguerrero@ece.buap.mx, \\ Nicolas.Marchand@gipsa-lab.grenoble-inp.fr, guylaine.poulin-vittrant@univ-tours.fr
}

\begin{abstract}
The paper deals with the development of a bounded control law for Flappingwing Micro Aerial Vehicles (FMAVs) that mimics a strategy adopted by animal flapping flyers to stabilize their orientation. The control consists on generating torques about the body's principal axes by means of a modulation of the wing angle amplitudes. It is known that flapping flyers orient their body without any numerical computation or estimation of their current attitude. Therefore, the proposed control law is computed using the direct measurements of on-board sensors mimicking animal sensitive organs, more specifically the halteres, legs sensilla and magnetic sense. The technological equivalents of these biological sensors are respectively three rate gyros, a tri-axis accelerometer and a tri-axis magnetometer. Besides, the control signal is bounded to keep the wing angle amplitudes below the maximal values. Owing to its simplicity, this control law is suitable for applications where on-board computational resources are limited. The stability of the closed loop system is proved based on Lyapunov analysis and averaging theory. The effectiveness of the proposed control law is shown in simulations. The robustness with respect to external disturbances is also shown emphasizing the importance and need of the bounded control.
\end{abstract}

\section{Introduction}

Animal flapping flyers depict several techniques to achieve flight and maneuvers moving their wings, body and legs (Dudley 2002). Principally, these techniques get benefit of the wing morphology, aerodynamic effects, sensory and actuating systems, flight control and obstacle avoidance mechanisms. For example, insects are able to change, very quickly, their speed and direction of flight, within almost $100 \mathrm{~ms}$ especially during predatory phases (Dudley 2002). They are also able to accomplish a transitory lateral or backwards flight. These maneuvers are performed by a displacement of the abdomen or an asymmetrical evolution of the wings using an amplitude modulation (Nachtigall \& Wilson 1967; Alexander 1986) or phase modulation (Mountcastle \& Danie 2010), frequency modulation occurs very rarely (Nachtigall \& Wilson 1967).

Besides, the flapping flyer is endowed with information, issued from multiple sensitive organs, about its state, interaction with the environment as well as local destination to

$\dagger$ The paper lies within the scope of the project OVMI/EVA sponsored by the French National Research Agency. 


\section{H. Rifaï ${ }^{1}$, J.-F. Guerrero-Castellanos ${ }^{2}$, N. Marchand ${ }^{3}$, G. Poulin-Vittrant ${ }^{4}$}

determine the way to reach it. For insects, one can cite the ocelli, the compound eyes and other biological sensors detailed in the following (Dudley 2002; Alexander \& Vogel 2004; Campolo et al. 2009) (and references therein):

- Halteres: are gyroscopic biological sensors, present at the wing bases, that detect the rotational movement of the body and allow to determine its angular velocity along the three axes,

- Sensilla: are cuticular sense hair detecting chemical or mechanical stimuli. They are present on the antenna, wings and legs. Legs sensilla, for example, allow to determine the direction of the gravity field with respect to the insect's body,

- Magnetic sense allows to determine the direction of the earth magnetic field with respect to the insect's body.

Using a fusion of information issued from these organs, the insect determines its trajectory and adapts its body's velocity and orientation to track it. One should emphasize that these actions are executed by the insect without any instantaneous numerical knowledge of its position, orientation or velocity.

Insects performance has encouraged the design of Flapping-wing Micro Aerial Vehicles (FMAVs). The micro aerial vehicle is a small size aircraft, having a maximal dimension of 6 inchest, and intended to perform an autonomous flight, thanks to an onboard control system, comprising a set of sensors and a dedicated integrated circuits. Due to their small size and flapping movement, FMAVs fly in zones characterized by low Reynolds numbers $\left(10^{2}-10^{4}\right)$. They develop, therefore, extra non-stationary aerodynamic forces, which help them reduce the forces generated by the actuators to accomplish the flight and, consequently, reduce the energetic consumption. FMAVs produce low noise. They are able to accomplish vertical taking-off and landing as well as stationary flight in hovering mode. However, their major drawback is still the difficulty of identifying and implementing the complex mechanisms carried out by insects to perform maneuvers (Dudley 2002; Hedrick \& Daniel 2006). The FMAVs are intended for use in areas inaccessible for people or that require high accuracy of intervention.

The present paper deals with the modeling and attitude stabilization of a FMAV for scenarios necessitating to maintain a stable orientation in front of a scene in order to monitor or investigate it. A simple model mimicking the flapping flight is presented, including the wings degrees of freedom, the sensorimotor system as well as the developed aerodynamic forces. The major contribution of this work is the development of a biomimetically inspired control strategy aiming to stabilize the orientation of the FMAV. The inputs of the control law are the direct measurements of on-board sensors, equivalent to those which an insect is equipped with, without the need of an explicit attitude reconstruction (represented by the Euler angles in $\mathbb{R}^{3}$, quaternion in $\mathbb{S}^{3} \subset \mathbb{R}^{4}$ or rotation matrix in $S O(3)$ ). Hence, unlike conventional approaches, the computational cost used for the attitude estimation/reconstruction is avoided. Note that attitude estimation is frequently carried out by means of Extended Kalman Filters or Nonlinear observers which represent a high computational cost. The control law takes into consideration the wing angle amplitude bounds, characterizing the animal species. It has the easiness of a PD controller, the derivative term is obtained by means of the halteres (angular velocity) and the proportional term by means of legs sensilla and magnetic sense (direct measurement of attitude error). Moreover, the control is very simple and therefore is suitable to be implemented in real time. It is also independent of the FMAV body's inertia, modeling or aerodynamic errors and robust with respect to external disturbances. Unlike fuzzy

$\dagger$ The definition is given by the DARPA (Defense Advanced Research Projects Agency). 
controllers or fractional PID, the bounds of the control signal are taken into account explicitly and the stability properties are well established.

Models of FMAVs existing in literature consider them as rigid bodies subject to external forces and torques generated by the flapping wings whose amplitudes may be modulated (Deng et al. 2006a; Rakotomamonjy et al. 2010), phase or even both of them (Chung \& Dorothy 2010; Oppenheimer et al. 2010; Couceiro et al. 2012). Few works have treated the problem of controlling the orientation of flapping aerial vehicles based on sensor measurements. A proportional derivative output feedback is proposed in (Schenato et al. 2004) based on ocelli and halteres measurements. A linear quadratic optimal control is proposed in (Deng et al. 2003, 2006b) based on ocelli, magnetic compass and halteres measurements. In (Epstein et al. 2007), halteres measurements are used to estimate the pitch rate and stabilize the corresponding angle with a proportional derivative controller with pole placement. Rate gyros, accelerometer and magnetometer sensors, representing respectively the halteres, legs sensilla and magnetic compass, are used in (Campolo et al. 2009) to estimate the insect's attitude (rotation matrix), used after, along with angular velocity measurement, in a state feedback control law. One should emphasize that, in the aforementioned works, the proposed linear control laws cannot be sufficiently robust with respect to external disturbances representing wind for example. Note also that building a control law using estimated attitude state is not a biomimetic approach. In fact, insects have no numerical instantaneous determined orientation knowledge (angles or rotation matrix) but only information issued directly from their biological sensors (Chapman 1998).

The paper is organized as follows. In section 2, the model of the FMAV is proposed in agreement with insect models, including the sensorimotor systems, aerodynamic forces and body's dynamics. A biologically inspired bounded control law, based directly on the measurements of some embedded sensors and aiming to stabilize the orientation of the FMAV, is presented in section 3. Simulation results are addressed in section 4 as well as some robustness tests. Finally, conclusions are given in section 5 and future works are introduced.

\section{Insect flight versus biomimetic robot flight}

The present section deals with the design and modeling of a flapping-wing micro aerial vehicle. Design concerns mainly the choice of the sensors and actuators to embark on the FMAV. Therefore, the insect's sensorimotor system is shortly presented and correlated to the technological corresponding equipments. Modeling concerns principally the establishment of a simple mathematical model allowing to represent the degrees of freedom of the flapping flight as well as aerodynamic forces and mechanisms deployed by nature's flapping flyers to perform maneuvers. One should note that a beating wing is in interaction with the surrounding air flow, which creates aerodynamic forces perpendicular to its surface. These forces generate the linear and rotational movements of the FMAV's body.

\subsection{Wing degrees of freedom}

The movement of a wing is a combination of several elementary actions (Thomson et al. 2009; Chapman 1998): flapping, feathering, lagging or elevation besides flexion and torsion (FIGURE 1 and 2). Flapping is an up-and-down movement of the wing represented by a rotation of the wing about its tangential axis $\vec{t}$ of a flapping angle $\phi$. Feathering is a rotation of the wing about its span-wise axis $\vec{r}$ of a rotation angle $\psi$. Lagging or elevation is a forward-backward movement of the wing parallel to the body, modeled by a rotation about a normal axis $\vec{n}$ of a deviation or elevation angle $\theta$. Flexibility of the wing allows 

lagging axis

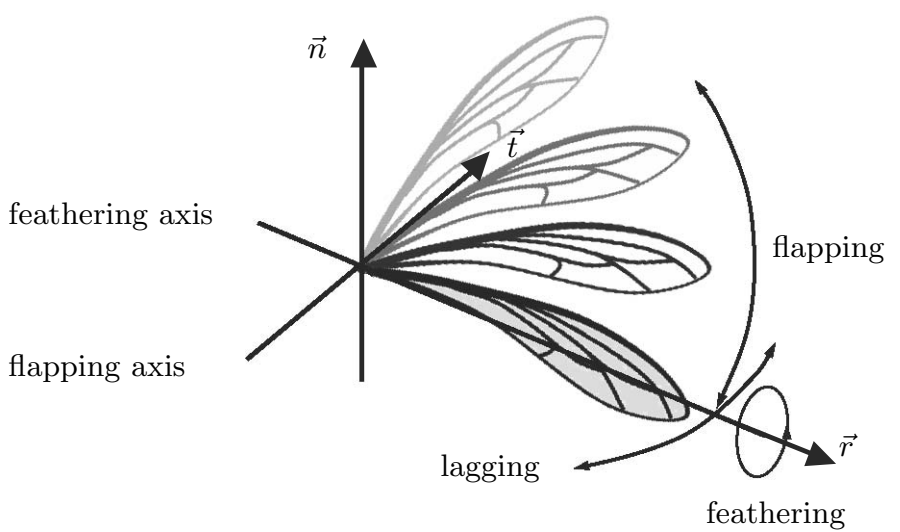

Figure 1. Degrees of freedom of a wing: flapping, lagging (elevation) and feathering

it to resist to turbulence, provides a gentler flight and increases the aerodynamic force relative to a same size rigid wing (Shyy et al. 2010). Torsion is a twist movement of the wing, providing an aerodynamic stability (Senda et al. 2008).

The directions of the wing rotational axes are chosen such that $\vec{r}$ is oriented from the wing base to its tip along the wingspan, $\vec{t}$ is parallel to the wing chord, oriented from trailing to leading edge and $\vec{n}$ is perpendicular to the wing plane oriented so that the three-sided frame $\mathcal{F}^{w}(\vec{r}, \vec{t}, \vec{n})$ is direct. Note that the frame $\mathcal{F}^{w}$ should be indexed, left $\mathcal{F}_{l}^{w}$ and right $\mathcal{F}_{r}^{w}$, relative to the left and right wings respectively.

Maneuvers of animal flapping flyers are performed primarily by asymmetrical movements of the wings, a displacement of the abdomen and legs. The movement of the wings generates aerodynamic forces, which resultant is perpendicular to the wing surface. Therefore, the flapping/rotation movements of the wings generate the vertical/longitudinal movements of the insect, respectively. A difference in the amplitudes of the left and right flapping/rotation angles allows to generate a roll/yaw movements, respectively (FIGURE 2). The pitch movement can be created by controlling the wing's elevation degree of freedom, or by changing the center of gravity of the body tilting it upward or downward. The second solution allows to reduce the number of embarked actuators and therefore is adopted in the sequel. Finally, the lateral movement is generated by coupling the body vertical lift force with the roll angle, maneuver performed by many birds and insect species (Dudley 2002).

The wing angles are characterized by their maximal amplitudes and their wingbeat frequency within their predefined trajectory. These values are specific to each species. Generally, flapping-wing flying creatures move their wings according to a sine function with higher harmonics, the fundamental frequency is equal to the wingbeat frequency (Dudley 2002). A wingbeat period is divided into two phases: the downward phase of the flapping movement or downstroke and, the upward phase or upstroke (Chapman 1998; Dudley 2002). During downstroke, the flapping flyer orients the dorsal side of the wing to the flow. It orients the ventral side during the upstroke. The model proposed in this work considers the wing as a rigid body, flapping in the mean stroke plane, defined by taking the deviation angle $\theta$ to zero. This choice is motivated by the possibility to control insects using only two wing angles (Lentink \& Biewener 2010). Flapping and rotation angles, $\phi$ and $\psi$, are assumed to vary according to saw tooth and pulse functions respectively, such that the wing changes its orientation at the end of each half stroke (FIGURE 3). 


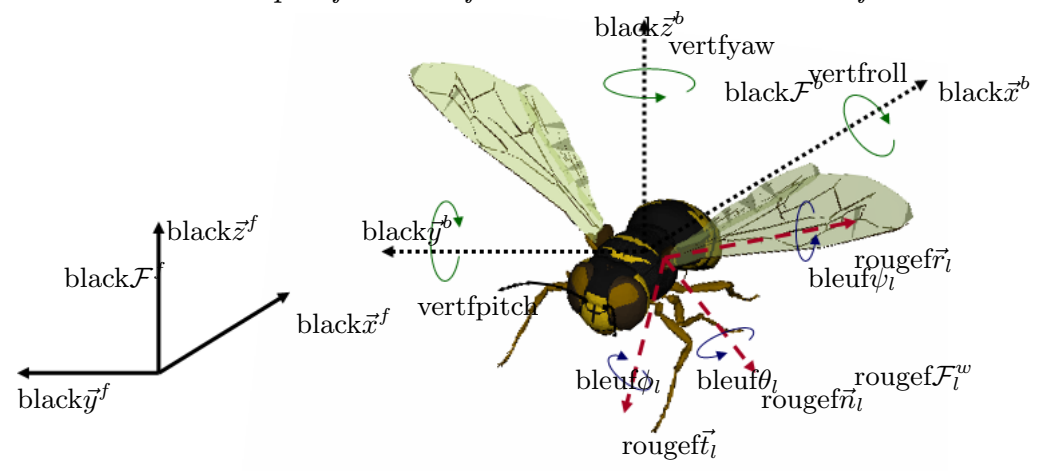

FIGURE 2. Coordinate frames: inertial fixed frame $\mathcal{F}^{f}\left(\vec{x}^{f}, \vec{y}^{f}, \vec{z}^{f}\right)$, body attached frame $\mathcal{F}^{b}\left(\vec{x}^{b}, \vec{y}^{b}, \vec{z}^{b}\right)$ and left wing attached frame $\mathcal{F}_{l}^{w}\left(\vec{r}_{l}, \vec{t}_{l}, \vec{n}_{l}\right)$

The time variation of the wing angles is given by (2.1):

$$
\begin{aligned}
& \phi(t)=\left\{\begin{array}{lr}
\phi_{0}\left(1-\frac{2 t}{\kappa T}\right) & 0 \leq t \leq \kappa T \\
\phi_{0}\left(2 \frac{t-\kappa T}{(1-\kappa) T}-1\right) & \kappa T<t \leq T
\end{array}\right. \\
& \psi(t)=\psi_{0} \operatorname{sign}(\kappa T-t) \quad 0 \leq t \leq T \\
& \theta(t)=0 \quad 0 \leq t \leq T
\end{aligned}
$$

where sign designates the classical sign function, $T$ is the wingbeat period, $\kappa$ is the ratio of downstroke duration to the wingbeat period and should verify $0<\kappa<0.5$ to produce a positive aerodynamic lift force over a wingbeat period, $\phi_{0}$ and $\psi_{0}$ are respectively the amplitudes of flapping and rotation angles. $\phi_{0}$ and $\psi_{0}$, considered for left and right wings, will define the control inputs in the following. This choice is motivated by the fact that wingbeats have generally fixed frequency, except during some maneuver phases. Turns can be created by asymmetrically changing the left and right wing angle amplitudes (Nachtigall \& Wilson 1967; Alexander 1986).

Note that the wingbeat frequency has been taken equal to $100 \mathrm{~Hz}$ considering in the following the model of a diptera insect (Dudley 2002). Note also that the given angle parametrization does not represent the real movement of the wings, but the desired trajectory to be achieved by the wing actuators.

Remark 1. The wing angle parametrization adopted in the present work is not unique. Other parameterizations can be used as well. The only condition is that they allow the generation of an aerodynamic body lift force that, averaged over a wingbeat period, should be positive and able to balance the FMAV's weight. The control strategy and control law proposed in the following remain always applicable.

Other non-symmetric wing-parametrizations have been proposed in the literature. In (Chung \& Dorothy 2010), all three angles have been modulated. However, embarking many actuators is weight costly. Therefore, strategies have been oriented to decrease their number. In (Oppenheimer et al. 2009), two actuators controlling the wings have been used besides a bobweight actuator in the body. In (Oppenheimer et al. 2010), the bobweight has been removed and the wings parametrization adapted to use only one actuator per wing. This configuration necessitates the development of complex control laws to stabilize the translational and rotational movement of the FMAV. In (Finio et al. 2009), three actuators have been introduced, one principal and two secondary to accomplish an asymmetric control of the wing angle amplitudes. Another work has considered one active and one passive degrees of freedom allowing to control four wing 

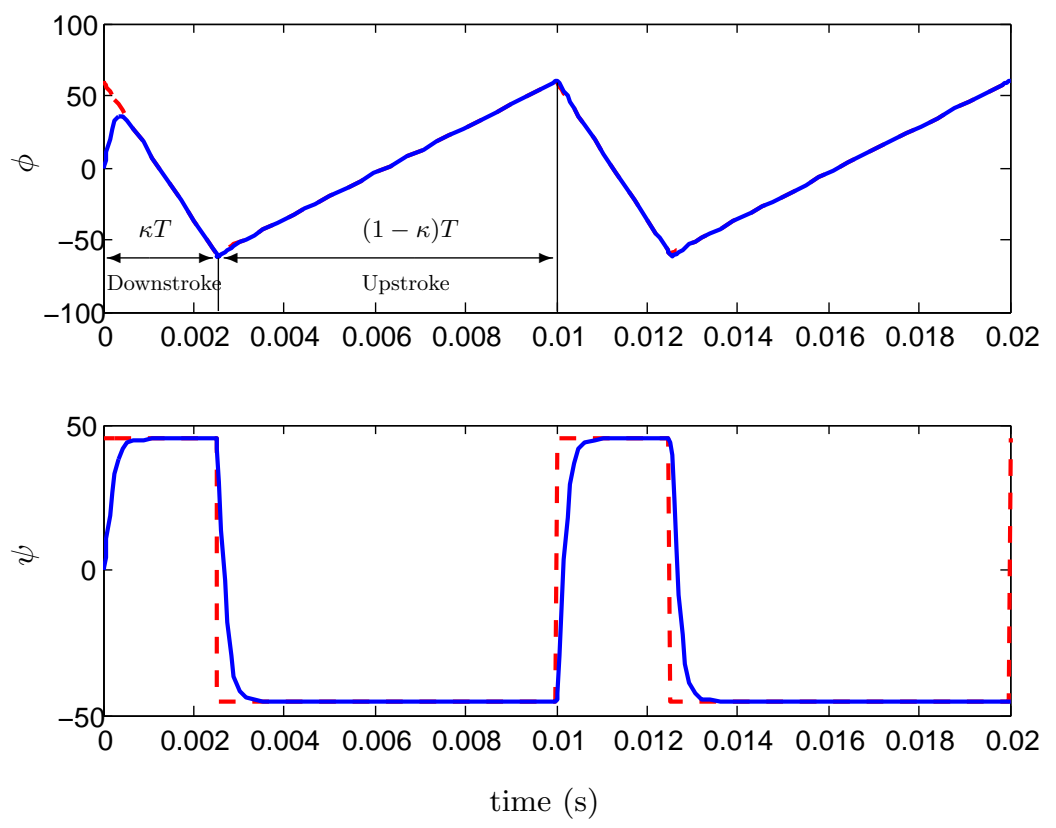

FIGURE 3. Shape of the wing angle evolution through an illustrative example: The flapping (top) and rotation (bottom) wing angles, the reference angles in dashed red line and the real angles (delivered by the actuators) in continuous blue line.

angles, the aim is to ensure the vertical flight, the orientation, notably the roll and yaw angles, is passively regulated (Sreetharan \& Wood 2011).

\subsection{Actuation}

The periodic wingbeats are accomplished by the muscles, present at the wing bases or in the thorax and, vibrating with a fixed frequency. This movement can be identified as a vibration at the undamped natural frequency. Therefore, piezoelectric actuators are the most suitable for this application. The reverse effect of piezoelectricity, consisting on applying voltage and retrieving mechanical movement, is of interest. The alternative voltage is delivered by an electronic converter designed specifically for piezoelectric actuators. These actuators behave as reactive loads (Janocha \& Stiebel 1998; Campolo et al. 2003) introducing some non-linearities (hysteresis, creep) that can be avoided using a local control (Kuhnen et al. 2006). The controller inputs are the reference angles defined in (2.1). The local closed loop of controller-actuator is regulated to behave as a first-order filter having fast dynamics so that the influence of the actuator on the global system dynamics is despised (Figure 3):

$$
\ddot{A}=\ddot{A}_{r}-\lambda_{1}\left(\dot{A}-\dot{A}_{r}\right)-\lambda_{2}\left(A-A_{r}\right)
$$

with $A$ the amplitude of the flapping or rotation angles at the actuator's output and $A_{r}$ is the reference amplitude at the actuator's input. $\lambda_{1}$ and $\lambda_{2}$ are computed using pole placement such that the time constant of the local closed loop is equal to $\tau=0.1 \mathrm{~ms}$ : $\lambda_{1}=\frac{2}{\tau}$ and $\lambda_{2}=\frac{1}{\tau^{2}}$.

Remark 2. Note that the piezoelectric actuators available on the market have very fast dynamics with a time response reaching the microsecond range (PZT piezoceramic actuators for example). The conservative hypothesis considered for $\tau=0.1 \mathrm{~ms}$ is therefore 
absolutely realistic even when the actuator is coupled to the wing. Note that if the time response of the wing-actuator system is less than $0.1 \mathrm{~ms}$, it will be more advantageous for the transparency of the actuator and its influence on the FMAV's movement.

\subsection{Sensory system}

In attitude stabilization theory, the FMAV's body should reach a desired orientation and maintain it, which means that, at stationary hovering flight, the body axes should be aligned to some reference axes with a null angular velocity. Within insect bio-sensors, the halteres, legs sensilla and magnetic sense contribute to control the body's orientation. These sensitive organs have some technological equivalents: respectively, the rate gyros, accelerometers and magnetometers.

Define a fixed frame in the space $\mathcal{F}^{f}\left(\vec{x}^{f}, \vec{y}^{f}, \vec{z}^{f}\right)$ and a mobile frame attached to the FMAV's body at its center of gravity $\mathcal{F}^{b}\left(\vec{x}^{b}, \vec{y}^{b}, \vec{z}^{b}\right)$ (indexes $f$ and $b$ stand for fixed and body, respectively) (FIgure 2). Define also a rotation matrix $R \in S O(3)=\left\{R \in \mathbb{R}^{3 \times 3}\right.$ : $R^{T} R=I_{3}$, det $R=1$ \} allowing the transformation from the fixed frame $\mathcal{F}^{f}$ to the body frame $\mathcal{F}^{b}$.

\subsubsection{Rate gyros}

Three rate gyros are mounted orthogonally on the FMAV's body such that their axes coincide with the body's axes. The sensors deliver the angular velocity measurements about the body's axes. The measured angular velocity, $\omega_{G}$, is given by:

$$
\omega_{G}=\omega+\xi_{G}
$$

where $\omega \in \mathbb{R}^{3}$ is the angular velocity of the body, $\xi_{G} \in \mathbb{R}^{3}$ is a white gaussian noise of the rate gyros.

\subsubsection{Magnetometer}

A tri-axis magnetometer is mounted on the FMAV such that its axes coincide with the body's axes. The sensor gives the measurement of the fixed magnetic field in $\mathcal{F}^{b}$ :

$$
s_{M}^{b}=R s_{M}^{f}+\xi_{M}
$$

where $s_{M}^{f} \in \mathbb{R}^{3}$ is the magnetic field in the fixed frame $\mathcal{F}^{f}, R$ is the rotation matrix from the fixed frame $\mathcal{F}^{f}$ to the body's frame $\mathcal{F}^{b}$ and $\xi_{M} \in \mathbb{R}^{3}$ is a vector of gaussian white noise. Note that the magnetic field in the fixed frame is considered constant and equal to $s_{M}^{f}=\left[\frac{1}{2}, 0,-\frac{\sqrt{3}}{2}\right]^{T}$ in the geographic zone where the simulations are performed.

\subsubsection{Accelerometer}

A tri-axis accelerometer is mounted on the FMAV such that its axes coincide with the body's axes. The sensor measures the FMAV's acceleration in $\mathcal{F}^{b}$. It is given by:

$$
s_{A}^{b}=R s_{A}^{f}+\xi_{A}
$$

where $s_{A}^{f}=\left(a-g e_{3}\right)$ with $e_{3}=[0,0,1]^{T}, g \in \mathbb{R}$ and $a \in \mathbb{R}^{3}$ are respectively the gravity and body's acceleration vectors expressed in the fixed frame $\mathcal{F}^{f} . R \in \mathbb{R}^{3 \times 3}$ is the rotation matrix from the fixed frame $\mathcal{F}^{f}$ to the body frame $\mathcal{F}^{b} . \xi_{A} \in \mathbb{R}^{3}$ is a gaussian white noise. Considering that the FMAV moves at low accelerations such that $\|a\| \ll\|g\|$ and normalizing, the accelerometer measurement is given by:

$$
s_{A}^{b}=R g_{n}+\xi_{A}
$$

with $g_{n}=s_{A}^{f}=[0,0,-1]^{T}$ the normalized gravity vector. 


\section{H. Rifaï ${ }^{1}$, J.-F. Guerrero-Castellanos ${ }^{2}$, N. Marchand $^{3}$, G. Poulin-Vittrant ${ }^{4}$}

Remark 3. The FMAV experiments acceleration along the body-fixed direction $\vec{z}^{b}$ for the taking-off, landing and translational horizontal movements. Because in the present work, only the attitude stabilization is considered, the assumption $\|a\| \ll\|g\|$ holds and the tri-axis accelerometer can be used as a reference vector sensor. The gravity vector is the reference vector and the acceleration is considered as a disturbance. If the translational movement has to be considered, a compensation of the vertical acceleration should be envisaged.

\subsection{Aerodynamics}

The flapping movement of the wings within a surrounding steady air flow generates quasisteady aerodynamic forces: a lift force, perpendicular to the wing, enhancing the flight and a drag force, parallel and opposing to the air flow (Dudley 2002). Drag forces generated by the wings are neglected in the present work considering that the wings are made of materials having a sufficiently small friction coefficient and that the drag is generated only by the body (Dudley 2002). In addition to the quasi-steady aerodynamic forces, the flapping flyer is subject to unsteady aerodynamic forces generated by the rotation of the wing about its radial axis $\vec{r}$. This rotation creates vortices, adding air flow to the following wingbeats, and creating additional forces. These forces allow the flapping-wing animal to ensure maneuvers such as quick variations of velocity or flight direction and instantaneous turns. All forces are considered applied at the wing's center of pressure, located at $l_{r}$ equal to $65 \%$ of the wing length $L$ from the wing base $\left(l_{r}=0.65 L\right)$ and at $l_{t}$ equal to $25 \%$ of the chord length $C_{h}$ from the leading edge $\left(l_{t}=0.25 C_{h}\right)$ (Schenato et al. 2003). The center of pressure is considered belonging to the radial axis $\vec{r}$, given in the wing frame $\mathcal{F}^{w}$ by $p^{w}=\left[l_{r}, 0,0\right]^{T}$.

\subsubsection{Quasi-steady aerodynamic force}

The quasi-steady force is generated by the pressure of the air flow exerted on the wing surface. This force is perpendicular to the wing and is applied at the wing's center of pressure. It is oriented to the opposite direction of the wing's velocity. The magnitude of this force is given by:

$$
f_{q s}=-\frac{1}{2} \rho C_{w} S_{w} v^{w}\left|v^{w}\right|
$$

with $\rho$ the air density, $S_{w}$ the wing surface and $v^{w}$ the wing velocity. $C_{w}$ is the aerodynamic coefficient of the wing. $C_{w}=C\left(1+C_{f}\right)$ during downstroke and $C_{w}=C\left(1-C_{f}\right)$ during upstroke, with $C \approx 3.5$ is the force coefficient derived empirically in (Dickinson et al. 1999; Schenato et al. 2003) and $C_{f}$ is a coefficient chosen so that the aerodynamic force is $20 \%$ greater during downstroke relative to upstroke. This dissymmetry emphasizes the fact that the convex dorsal side of the wing is oriented to the flow during the downstroke, while the concave ventral side of the wing is opposed to the flow during upstroke. The wing camber alteration is due to the stroke reversal of the air circulation around the wing, reducing the effective area of the wing (Dudley 2002). Therefore, the downstroke force is presumably higher than the upstroke one.

\subsubsection{Rotational force}

The rotation of the wing about its radial axis deviates the surrounding air flow. The wing reacts to this phenomenon by creating additional rotational circulation (Sane 2003) and consequently a rotational force modeled by (Rakotomamonjy et al. 2010):

$$
f_{r}=\pi \rho l_{r} C_{h}^{2}\left(\frac{3}{4}-\frac{l_{t}}{C_{h}}\right) v^{w} \dot{\psi}
$$


with $\dot{\psi}$ the first derivative of the rotation angle.

\subsubsection{Added mass force}

The added mass phenomenon is created by the acceleration of the additional fluid mass surrounding the wing when it accelerates and rotates. It can be modeled by (Rakotomamonjy et al. 2010):

$$
f_{m}=\frac{\pi}{4} \rho L l_{r} C_{h}^{2} \ddot{\phi}
$$

with $\ddot{\phi}$ the second derivative of the flapping angle.

In addition to these forces, the wing is subject to other phenomena like the wake capture, delayed stall, wagner effect, etc. that have a minor contribution to the total wing lift force and are difficult to model (Sane 2003).

The aerodynamic force generated by a wing is applied at its center of pressure, has the direction of the normal vector of the wing $\vec{n}$. Its expression in the wing's frame $\mathcal{F}^{w}$ is given by:

$$
f^{w}=f_{q s}+f_{r}+f_{m}
$$

The total aerodynamic force $f^{b} \in \mathbb{R}^{3}$, generated by the left and right wings, expressed in the body frame $\mathcal{F}^{b}$ is given by

$$
f^{b}=R_{l}^{b} f_{l}^{w}+R_{r}^{b} f_{r}^{w}
$$

with $R_{l, r}^{b}$ are the rotation matrices from the left or right wing frames to the body frame.

The aerodynamic torque expressed in the body frame is given by

$$
\tau^{b}=p_{l}^{b} \times f_{l}^{b}+p_{r}^{b} \times f_{r}^{b}
$$

with $p_{l, r}^{b}$ is the position of the left or right wing aerodynamic center in the body frame computed by $p_{l, r}^{b}=R_{l, r}^{b} p^{w}$. It is considered, in the present work, that the aerodynamic and pressure centers coincide.

The left and right wing velocities, expressed in the body frame, are computed by deriving the wing center of pressure positions $v_{l, r}^{b}=\dot{p}_{l, r}^{b}$. Their projections in the wing frames are obtained by a simple rotation $v_{l, r}^{w}=R_{b}^{l, r} v_{l, r}^{b}$.

\subsection{Body's dynamics}

The body can be considered as a whole entity and is modeled as a rigid body, to which are attached two wings. The wing inertia is neglected in the present work because their mass is less than $5 \%$ of the body's mass (Schenato et al. 2003). The effect of the wings' inertia due to the high flapping frequency is considered beyond the scope of this paper. Therefore, the FMAV is modeled as a rigid body subject to aerodynamic forces and torques that generate its movement. It is subject also to viscous and gravitational forces.

The rotational kinematics and dynamics of the FMAV are given by $(2.12,2.13)$. The translational dynamics are not presented here for sake of simplicity. The aerodynamic lift force balances the gravity effect in order to stabilize the FMAV in hovering mode. For more details, readers can refer to previous works (Rifai et al. 2008).

$$
\begin{aligned}
\dot{R} & =R \omega^{\times} \\
\dot{\omega} & =J^{-1}\left(\tau^{b}-\omega^{\times} J \omega\right)
\end{aligned}
$$

$\omega \in \mathbb{R}^{3}$ is the angular velocity with respect to the mobile frame $\mathcal{F}^{b} . \tau^{b} \in \mathbb{R}^{3}$ is the aerodynamic torque vector defined in $\mathcal{F}^{b} . J \in \mathbb{R}^{3 \times 3}$ is the inertia matrix of the body relative to $\mathcal{F}^{b}$. $R$ is the rotation matrix from the fixed frame $\mathcal{F}^{f}$ to the mobile frame 
$\mathcal{F}^{b} \cdot \omega^{\times}$is the skew symmetric matrix associated to the vector $\omega$ and related to the cross product $\times$ such that $\omega^{\times} a=\omega \times a$, with $a \in \mathbb{R}^{3}$.

\subsection{Control strategy}

Aerodynamic forces are function of the wing angles, their derivatives and some geometric and aerodynamic parameters. Controlling the orientation of the FMAV amounts from controlling the amplitude or the frequency of the wing angles. Considering that the nature's flapping flyers use generally a fixed characteristic frequency except during instantaneous maneuvers execution (Dudley 2002; Nachtigall \& Wilson 1967), the wing angle amplitude control is considered.

On the other hand, the wingbeat frequency adopted in the present work is relatively high $(100 \mathrm{~Hz})$. Aerodynamic forces and torques, which are generated at the wingbeat frequency, affect the body's movement only by their average values, computed over a wingbeat period. This is proved based on the averaging theory (Khalil 2002; Bullo 2002; Vela 2003) which is a strategy usually used in FMAVs control (Schenato et al. 2003).

Denote by $u=\left(\phi^{l}(t), \phi^{r}(t), \psi^{l}(t), \psi^{r}(t)\right)$ the flapping and rotation angles for left and right wings, $v=\left(\phi_{0}^{l}, \phi_{0}^{r}, \psi_{0}^{l}, \psi_{0}^{r}\right)$ the amplitudes of these angles (2.1), then $u=v f_{2}(t)$. Denote also by $x=(R, \omega)$ the FMAV's rotational state, the model given by $(2.12-2.13)$ can be written in a compact form as:

$$
\dot{x}=f_{1}(x, u)
$$

Let $\bar{x}=(\bar{R}, \bar{\omega})$ denote the averaged state over a wingbeat period $T$. The compact form of the FMAV's model, averaged over a wingbeat period, is given by:

$$
\dot{\bar{x}}=\bar{f}_{1}(\bar{x}, v)
$$

Averaging theory shows that an exponential stable equilibrium state for the averaged dynamics of a high frequency oscillating system $\left(\bar{x}=x_{e}\right)$ is also a stable equilibrium state for the oscillating (time variant) system: there exists $k>0$ such that $\|x(t) \otimes \bar{x}(t)\|<k T$ for all $t \in[0, \infty)$, with $\otimes$ designates a comparison operator and $T$ the wingbeat period which is small in the present case.

As mentioned previously, the FMAV is controlled indirectly by means of the wing angle amplitudes $v$ that will be computed using a feedback $h(\cdot)$ of direct sensors measurements $\omega$ and $s_{k}, k \in\{1, \ldots, n\}$ with $n$ the number of on-board reference sensors:

$$
v=h\left(\omega, s_{k}\right),
$$

Using the inverse reasoning, the control strategy is defined as follows. On one hand, based on the desired orientation and the current one measured by on-board sensors, a control torque is computed. This control is actually equal to the average torque $\bar{\tau}$ over a wingbeat period: $\bar{\tau}=\mathcal{U}\left(\omega, s_{k}\right)$. On the other hand, based on the expression of the aerodynamic torque, a relation between the mean torque $\bar{\tau}$ and the wing angle amplitudes is determined. Recall that the flapping and rotation angle amplitudes $\left(\phi_{0}, \psi_{0}\right)$ are the control inputs. Therefore: $\bar{\tau}=\Lambda\left(\phi_{0}, \psi_{0}\right)$. The inverse relation can be used to compute the wing angle amplitudes, that should be applied at the beginning of a wingbeat period, function of the control torque: $\left(\phi_{0}, \psi_{0}\right)=\Lambda^{-1}(\bar{\tau})=\Lambda^{-1}\left(\mathcal{U}\left(\omega, s_{k}\right)\right)$. Therefore, $h(\cdot)=\Lambda^{-1}(\mathcal{U}(\cdot))$.

Two actuators are used for each wing to generate the flapping and rotation angles, creating then the roll and yaw movements of the FMAV. The pitch movement is generated by moving a small mass inside the body using the ElectroWetting On Dielectric (EWOD) technology (Renaudin et al. 2004). A similar strategy using a bobweight actuator inside the FMAV has also been adopted in (Oppenheimer et al. 2009). 
Remark 4. Analyzing only the rotational movement of the FMAV, the system can be considered as fully actuated. However, when studying the translational movement, the system will become underactuated and a coupling of the rotational and translational dynamics should be performed to execute a three dimensional movement. Hence arises the importance of the attitude control.

Computing the averaged roll and yaw torques, function of the flapping and rotation angle amplitudes, one has the explicit form of the trigonometric function $\Lambda(\cdot)$ :

$$
\begin{aligned}
& \bar{\tau}_{1}=\beta l_{r}\left[\phi_{0}^{r^{2}} \cos \psi_{0}^{r}-\phi_{0}^{l^{2}} \cos \psi_{0}^{l}\right. \\
& \bar{\tau}_{3}=\alpha l_{r}\left[\phi_{0}^{r^{2}} \sin \psi_{0}^{r}-\phi_{0}^{l^{2}} \sin \psi_{0}^{l}\right]
\end{aligned}
$$

with

$$
\begin{aligned}
\alpha & =\frac{2}{T^{2}} \frac{1+(1-2 \kappa) C_{f}}{\kappa(1-\kappa)} \rho C S_{w} l_{r}^{2} \\
\beta & =\frac{2}{T^{2}} \frac{1-2 \kappa+C_{f}}{\kappa(1-\kappa)} \rho C S_{w} l_{r}^{2}
\end{aligned}
$$

Each species is characterized by maximum flapping and rotation angles. In a practical point of view, this represents also a technological constraint because the actuators inputs should be bounded to avoid their saturation. Therefore:

$$
\begin{aligned}
& 0 \leq \phi_{0} \leq \phi_{0_{\max }} \\
& 0 \leq \psi_{0} \leq \psi_{0_{\max }}
\end{aligned}
$$

The roll and yaw torques are therefore bounded. Note also that the movement of the mass inside the body is limited by the body length, the mass and velocity used within the EWOD technology, therefore, the pitch control is bounded too. Resuming, $\bar{\tau} \in$ $\left[-\tau_{\max }, \tau_{\max }\right]$.

\section{Biologically inspired attitude stabilization}

The attitude stabilization problem consists in reaching a desired orientation and maintaining it all over the time. The error between the desired orientation defined by $R_{d}$ and the current orientation defined by $R$ is denoted by $R_{e}=R R_{d}^{T}$. Therefore, the attitude stability condition is expressed as:

$$
\left\{\begin{array}{lll}
\omega & \rightarrow & 0 \\
R_{e} & \rightarrow & I_{3}
\end{array}\right.
$$

with $I_{3}$ the three dimensional identity matrix. The angular velocity can be directly accessed based on the measurements of the rate gyros. However, no measurement of the rotation matrix can be obtained without using estimation strategies. Therefore, a biologically inspired technique is used in this work. It consists on defining the attitude error by means of the FMAV's on-board sensors which are equivalent to the animal flapping flyers' sensitive organs as it was described earlier. It is actually equal to the error between the on-board sensor measurements and the desired measurements without any computation of the body's orientation. Let $s_{k}^{b}$ and $s_{k}^{f}, k \in\{1, \ldots, n\}$, denote the measurement of the on-board sensor $k$ in the body frame $\mathcal{F}^{b}$ and fixed frame $\mathcal{F}^{f}$, respectively, with $n$ the total number of the embarked reference sensors. The projections of the unit vector $\vec{s}_{k}$ in the body and fixed frames are linked through the rotation matrix $R$ such as $s_{k}^{b}=R s_{k}^{f}$. Note that $s_{k}^{b}$ is called vector observation and $s_{k}^{f}$ reference vector. The attitude error $\gamma$ is 
defined as:

$$
\gamma=\frac{1}{n} \sum_{k=1}^{n}\left(R s_{k}^{f}\right) \times\left(R_{d} s_{k}^{f}\right)=\frac{1}{n} \sum_{k=1}^{n} s_{k}^{b} \times R_{d} s_{k}^{f}
$$

Reaching the desired attitude is expressed by a rotation matrix $R$ equal to the desired one $R_{d}$, i.e. relative to the accessible information $\gamma=0$. Reversely, $\gamma=0$ means that $s_{k}^{b}$ and $R_{d} s_{k}^{f}$ are collinear (3.1). Since $\vec{s}_{k}$ is a unit vector, then $s_{k}^{b}= \pm R_{d} s_{k}^{f}$. Two cases can be identified: i) $s_{k}^{b}=R_{d} s_{k}^{f}$ i.e. the vectors observation have the same orientation of the reference vectors desired direction in $\mathcal{F}^{b}$ which defines the final goal to reach, and ii) $s_{k}^{b}=-R_{d} s_{k}^{f}$ i.e. the vectors observation have the opposite direction of the desired reference vectors direction $\mathcal{F}^{b}$. Therefore, they are defined by a rotation of $180^{\circ}(\pi \mathrm{rad})$ with respect to a plane, axis or point. The symmetry surface depends actually on the number of reference sensors or more precisely on the number of non-collinear reference vectors $n$ such that $k \in\{1, \ldots, n\}$. Three cases are analyzed:

a) $n=1$ : If only one vector observation is afforded, it is linked to the reference vector by means of the rotation matrix $R$ as $s_{1}^{b}=R s_{1}^{f}$ providing a two-dimensional constraint and unveiling any rotation about the axes $s_{1}^{b}$ or $s_{1}^{f}$, in the body and fixed frames respectively. Therefore, at least two different sensor measurements should be provided instantaneously to have complete information about the attitude error (Markley et al. 2006): $n \geq 2$. The case $n=1$ is therefore excluded.

b) $n=2$ : The symmetry surface is limited to an axis $\vec{n}$ perpendicular to the plane containing the two vectors $R_{d} s_{k}^{f}, k \in\{1,2\}$, at the vectors intersection. Therefore, $s_{k}^{f}=$ $-R_{d} s_{k}^{f}=R_{s} R_{d} s_{k}^{f}$ with $R_{s}$ the rotation matrix defining the symmetry, i.e. the rotation matrix about the axis $\vec{n}$ of $180^{\circ}$. In the specific case where $R_{d} s_{k}^{f}$ belong to one of the basis planes $\left(e_{1}^{f}, e_{2}^{f}\right),\left(e_{1}^{f}, e_{3}^{f}\right)$ or $\left(e_{2}^{f}, e_{3}^{f}\right)$, then the rotation is performed respectively with respect to the axes $\vec{e}_{3}^{f}, \vec{e}_{2}^{f}$ and $\vec{e}_{1}^{f}$. These special cases chiefly facilitate the problem and therefore, a choice of the reference sensors should be done this way. This technique is adopted in the present paper. The matrix $R_{s}$ is given by:

$$
R_{s} \in\{\operatorname{diag}(-1,1,1), \operatorname{diag}(1,-1,1), \operatorname{diag}(1,1,-1)\}
$$

c) $n \geq 3: s_{k}^{b}=-R_{d} s_{k}^{f}=S R_{d} s_{k}^{f}$ with $S$ the reflexion matrix defined by $\operatorname{diag}(-1,-1,-1)$. Note that $S$ is not a rotation matrix because $\operatorname{det} S=-1$ and $\operatorname{Tr} S=-3$. No rotation matrix can therefore be identified and the configuration $s_{k}^{b}=-R_{d} s_{k}^{f}$ cannot be reached.

Resuming, the set of couples $(\omega, R)$ for which the attitude stability is reached, ( $\omega=$ $0, \gamma=0)$, is given by:

$$
\mathcal{E}=\left\{(\omega, R) \in T S O(3): \omega=0, R=\mathcal{P} R_{d}\right\}
$$

with

$$
\mathcal{P}=\{\operatorname{diag}(1,1,1), \operatorname{diag}(-1,1,1), \operatorname{diag}(1,-1,1), \operatorname{diag}(1,1,-1)\}
$$

The block diagram of the closed loop attitude control is given in Figure 4 where the different blocks have been defined previously. In the following, the control torques will be addressed. The control forces are set such that they balance the gravity and ensure no movement along the three axes of the fixed frame $\mathcal{F}^{f}$.

\subsection{Bounded attitude control}

A bounded control torque computed using the direct measurements of on-board sensors is proposed. This control takes into consideration the saturation of the actuators and is simple to implement. First, the asymptotical stability of the rigid body is shown, then 


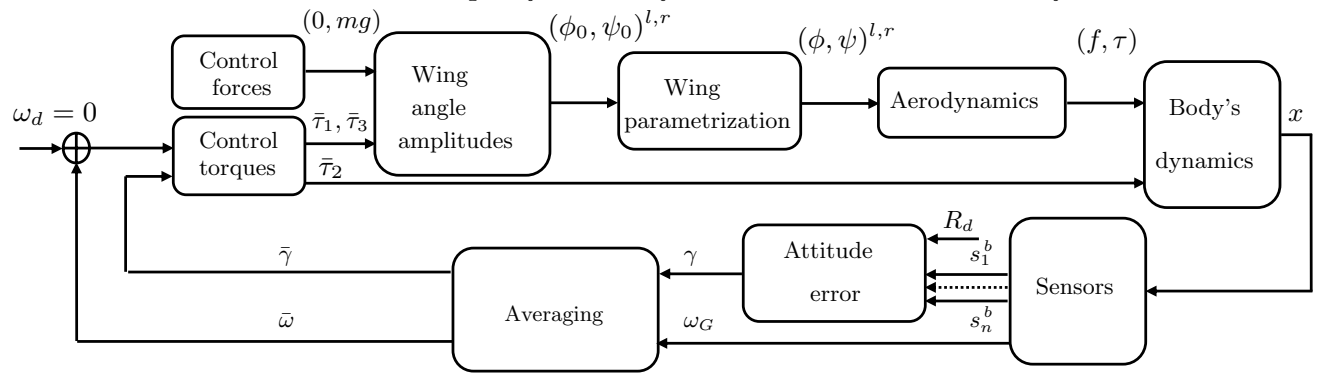

FIgURE 4. The block diagram of the FMAV's attitude control based on sensors measurements.

the proof is extended to show the stability of the FMAV. It is assumed that there exist at least two non-collinear vector observations embarked on the FMAV such that $n \geq 2$.

Proposition 1. Consider the flapping micro-aerial vehicle's rotational dynamics described by (2.12-2.13). The attitude error $\gamma$ between current and desired body's orientations is defined by (3.1) with $n \geq 2$. The FMAV body's angular velocity $\omega=\left[\omega_{1}, \omega_{2}, \omega_{3}\right]^{T}$ is measured by three rate gyros. Define the bounded control $\bar{\tau}=\left[\bar{\tau}_{1}, \bar{\tau}_{2}, \bar{\tau}_{3}\right]^{T}$ by:

$$
\bar{\tau}_{j}=-\operatorname{sat}_{N_{j}}\left(\frac{\lambda \bar{\omega}_{j}}{\rho_{j}}+\lambda \bar{\gamma}_{j}\right), \quad j \in\{1,2,3\}
$$

with $\bar{\omega}, \bar{\gamma}$ are respectively the averaged angular velocity and attitude error computed over a wingbeat period $T, N_{j}$ is the bound of the control torque component $\bar{\tau}_{j}$, sat s $_{j}(\cdot)$ is a classical saturation function, $\lambda$ is a positive real parameter such that $0<\lambda \leq \min \left(N_{j} / 2\right)$ and $\rho_{j}, j \in\{1,2,3\}$, are positive scaling parameters. The control torque (3.5) stabilizes the FMAV at $(\omega, R)=\left(0, R_{d}\right)$ with a domain of attraction equal to $T S O(3) \backslash\left\{\left(0, R_{s} R_{d}\right)\right\}$, $R_{s}$ is given in (3.2).

Proof. Let's first prove the stability of a rigid body whose state vector is the averaged state vector of a flapping FMAV. The dynamics of the rigid body are then given by (2.12-2.13) where the angular velocity is denoted by $\bar{\omega}$, the rotation matrix by $\bar{R}$, the control torque by $\bar{\tau}$ and the attitude error by $\bar{\gamma}$. Note that the derivative of an averaged vector observation $\bar{s}_{k}^{b}$ is linked to the vector by means of

$$
\dot{\bar{s}}_{k}^{b}=\bar{s}_{k}^{b} \times \bar{\omega}=-\bar{\omega} \times \bar{s}_{k}^{b}
$$

Note also that the reference vectors are well known and fixed vectors; the average over a wingbeat period is equal to the vector.

Consider first the following definite positive Lyapunov function:

$$
V=\frac{1}{2} \bar{\omega}^{T} J \bar{\omega}
$$

$J$ is the inertia matrix of the body. $V$ is trivially positive definite and radially unbounded. The derivative of (3.7) along the trajectories of the closed-loop system is given by

$$
\dot{V}=\bar{\omega}^{T} J \dot{\bar{\omega}}=\underbrace{\bar{\omega}^{T}\left(-\bar{\omega}^{\times} J \bar{\omega}\right)}_{=0}+\bar{\omega}^{T} \bar{\tau}=\bar{\omega}^{T} \bar{\tau}=\sum_{j=1}^{3} \bar{\omega}_{j} \bar{\tau}_{j}
$$

One gets from $\bar{\tau}_{j}$ in $(3.5)$ and (3.8) that 


$$
\dot{V}=-\sum_{j=1}^{3} \bar{\omega}_{j} \operatorname{sat}_{N_{j}}\left(\frac{\lambda \bar{\omega}_{j}}{\rho_{j}}+\lambda \bar{\gamma}_{j}\right)
$$

Let $\Phi=\{\bar{\omega}:|\bar{\omega}| \leq \rho+\varepsilon\}$ for some $\varepsilon>0$ and $\rho=\left[\rho_{1}, \rho_{2}, \rho_{3}\right]^{T}$. Outside $\Phi$, i.e. $\left|\bar{\omega}_{j}\right|>\rho_{j}+\varepsilon$, using the unitary condition $\left|\gamma_{j}\right| \leq 1$, it follows that $\left|\lambda \frac{\bar{\omega}_{j}}{\rho_{j}}+\lambda \bar{\gamma}_{j}\right| \geq \lambda \frac{\varepsilon}{\rho_{j}}$ and that $\lambda \frac{\bar{\omega}_{j}}{\rho_{j}}+\lambda \bar{\gamma}_{j}$ and $\bar{\omega}_{j}$ have the same sign. Therefore,

$$
\dot{V}=-\sum_{j=1}^{3} \bar{\omega}_{j} \operatorname{sat}_{N_{j}}\left(\frac{\lambda \bar{\omega}_{j}}{\rho_{j}}+\lambda \bar{\gamma}_{j}\right) \leq-\sum_{j=1}^{3} \frac{\lambda \varepsilon}{\rho_{j}}\left|\bar{\omega}_{j}\right|<-\sum_{j=1}^{3} \frac{\lambda \varepsilon\left(\rho_{j}+\varepsilon\right)}{\rho_{j}}<-3 \lambda \varepsilon<0
$$

Consequently, $\bar{\omega}$ enters in $\Phi$. During this time, $\bar{\gamma}_{j}$ cannot diverge since it is structurally unitary and therefore bounded.

Once in $\Phi$, one has:

$$
\left|\frac{\lambda \bar{\omega}_{j}}{\rho_{j}}+\lambda \bar{\gamma}_{j}\right| \leq 2 \lambda+\frac{\lambda \varepsilon}{\rho_{j}}
$$

Taking $\varepsilon$ sufficiently small and using the assumption that $2 \lambda<N_{j}$, then:

$$
\left|\frac{\lambda \bar{\omega}_{j}}{\rho_{j}}+\lambda \bar{\gamma}_{j}\right| \leq N_{j}
$$

Consequently, sat $_{N_{j}}$ operates in a linear region, and the control torque becomes:

$$
\bar{\tau}_{j}=\frac{\lambda}{\rho_{j}}\left(\bar{\omega}_{j}+\rho_{j} \bar{\gamma}_{j}\right)
$$

In $\Phi$, consider the Lyapunov function $W$ defined by:

$$
W=\frac{1}{2} \bar{\omega}^{T} J \bar{\omega}+\frac{\lambda}{n} \sum_{k=1}^{n}\left[1-\left(\bar{R} s_{k}^{f}\right)\left(R_{d} s_{k}^{f}\right)\right]
$$

$W$ is a continuous and positive definite function on $\operatorname{TSO}(3)$ because $W>0$ and $W(0, R d)=$ 0 . Since $\bar{s}_{k}^{b}=\bar{R} s_{k}^{f}$ and $s_{k}^{f}$ constant, the derivative of (3.10) is given by:

$$
\dot{W}=\bar{\omega}^{T} J \dot{\bar{\omega}}-\frac{\lambda}{n} \sum_{k=1}^{n}\left(\dot{\bar{s}}_{k}^{b} R_{d} s_{k}^{f}\right)
$$

By means of $(3.1,3.6)$ and the fact that $\bar{\omega}^{T}\left(-\bar{\omega}^{\times} J \bar{\omega}\right)=0,(3.11)$ becomes:

$$
\begin{aligned}
\dot{W} & =\bar{\omega}^{T} \bar{\tau}+\frac{\lambda}{n} \sum_{k=1}^{n} \bar{\omega}^{T}\left(\bar{s}_{k}^{b} \times R_{d} s_{k}^{f}\right) \\
& =\bar{\omega}^{T} \bar{\tau}+\lambda \bar{\omega}^{T} \bar{\gamma} \\
& =\sum_{j=1}^{3}\left(\bar{\omega}_{j} \bar{\tau}_{j}+\lambda \bar{\omega}_{j} \bar{\gamma}_{j}\right)=\sum_{j=1}^{3} \dot{W}_{j}
\end{aligned}
$$


Analyzing $\dot{W}_{j}$ for $j \in\{1,2,3\}$, one gets from $\bar{\tau}_{j}$ in (3.9) and (3.12) that:

$$
\begin{aligned}
\dot{W}_{j} & =-\bar{\omega}_{j}\left(\frac{\lambda \bar{\omega}_{j}}{\rho_{j}}+\lambda \bar{\gamma}_{j}\right)+\lambda \bar{\omega}_{j} \bar{\gamma}_{j} \\
& =-\frac{\lambda}{\rho_{j}} \bar{\omega}_{j}^{2}
\end{aligned}
$$

and

$$
\dot{W}=\sum_{j=1}^{3} \dot{W}_{j}=-\lambda \sum_{j=1}^{3} \frac{\bar{\omega}_{j}^{2}}{\rho_{j}} \leq 0
$$

The derivative of the Lyapunov function $W$ is negative semidefinite. Recall that $\operatorname{TSO}(3)$ is compact. Therefore, for any initial condition $(\bar{\omega}(0), \bar{R}(0)) \in T \operatorname{SO}(3)$, the set:

$$
\Omega=\{(\bar{\omega}, \bar{R}) \in T \operatorname{SO}(3): W(\bar{\omega}, \bar{R}) \leq W(\bar{\omega}(0), \bar{R}(0))\}
$$

is a compact, positively invariant set of the closed-loop. From LaSalle Invariance Principle, it follows that all solutions that start in $\Omega$ converge to the largest invariant subset of $\bar{\Omega}$ belonging to $\Omega \cdot \dot{W}(\bar{\omega}, \bar{R}) \equiv 0$ implies $\bar{\omega} \equiv 0$, then, substituting this last identity into the closed-loop system $(2.12,2.13,3.5)$, one has

$$
\bar{\Omega}=\{(\bar{\omega}, \bar{R}) \in T \operatorname{SO}(3): \bar{\omega} \equiv 0, \bar{\gamma} \equiv 0\}
$$

with $\bar{\gamma}$ defined in (3.1) over the averaged measurements. Then, the largest invariant subset of $\bar{\Omega}$ is given by $\mathcal{E}(3.3,3.4)$.

Therefore, $\mathcal{E}$ defines the set of equilibrium points for which the Lyapunov function $W$ (3.10) represents a minimum $(W=0)$ and a local maximum $(W=2 \lambda)$ corresponding respectively to the rotation matrices $\bar{R}=R_{d}$ and $\bar{R}=R_{s} R_{d}$ with $R_{s}$ given in (3.2). If the system is at one of these points at $t_{0}=0$, it will remain there for all $t \geq t_{0}$. The control law acts then to ensure the convergence of the closed-loop solutions, whose initial conditions do not verify $(\bar{\omega}, \bar{R})=\left(0, R_{s} R_{d}\right)$, to the stable equilibrium point given by $(\bar{\omega}, \bar{R})=\left(0, R_{d}\right)$ and corresponding to $W=\dot{W}=0$.

Therefore, the rigid body is asymptotically stable with a domain of attraction equal to $\operatorname{TSO}(3) \backslash\left\{\left(0, R_{s} R_{d}\right)\right\}$.

Once the asymptotic stability of the rigid body proven, the stability of the FMAV subject to the control torque (3.5) and computed over the averaged dynamics of the system $(\bar{\omega}, \bar{R})$ will be presented. For that, the averaging theory recalled in $\S 2.6$ is used. It states that for a high frequency oscillating system, the averaged and time varying dynamics are very close and therefore, a stable equilibrium of the averaged dynamics is also a stable equilibrium of the time varying dynamics: $\|\omega-\bar{\omega}\|<k_{1} T$ and $\left\|R \bar{R}^{T}\right\|<k_{2} T$ for $k_{1,2}>0$ and $T$ the wingbeat period. In other words, the state vector $(\omega, R)$ of the FMAV, subject to the control torque (3.5), converges to the equilibrium point $\left(0, R_{d}\right)$ with a domain of attraction equal to $\operatorname{TSO}(3) \backslash\left\{\left(0, R_{s} R_{d}\right)\right\}$.

\section{Simulations and robustness tests}

Two strategies have been adopted in the literature to test the control laws developed for animal flapping flyers. The first is based on models of the flapping flight including aerodynamics, body and wing dynamics (Deng et al. 2006a; Rakotomamonjy et al. 2010; Oppenheimer et al. 2010; Couceiro et al. 2012). Note that well developed models of flapping flight do not exist because the aerodynamics at low Reynolds numbers have not been yet perfectly identified (Dudley 2002). The second strategy consists on testing the 


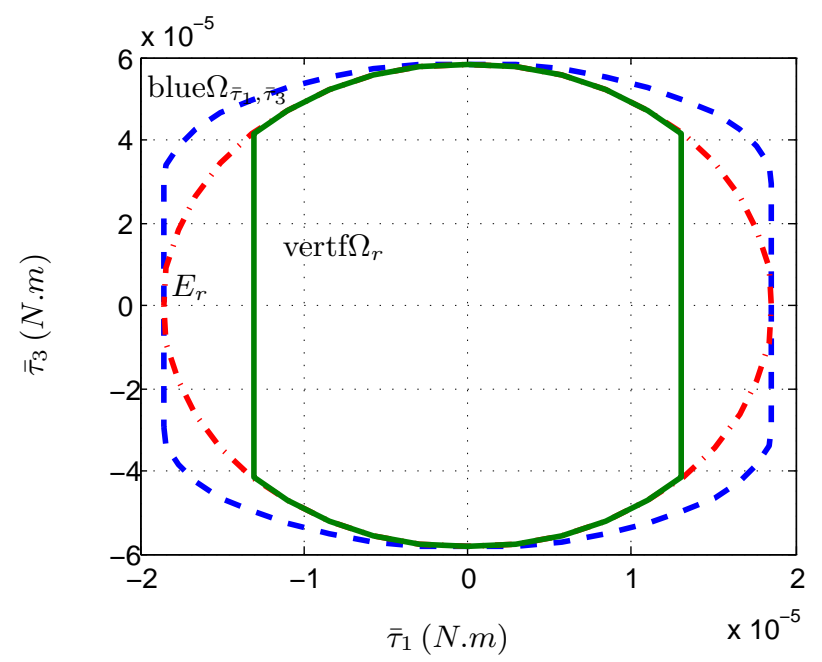

FiguRE 5. Yaw torque versus roll torque, defining the saturation set $\Omega_{\bar{\tau}_{1}, \bar{\tau}_{3}}$ approximated to an ellipse $E_{r}$ then to a set $\Omega_{r}$.

control in real time on a prototype (Finio et al. 2011; Perez-Arancibia et al. 2011) where simple models are proposed and identified. One should emphasize that at microscopic scale, only the vertical movement has been performed on a flapping flyer prototype (PerezArancibia et al. 2011) where the pitch movement is avoided by attaching the FMAV using some wires, the laser sensor measuring the vertical altitude is external as well as the power system. Vertical movement control of a $101 \mathrm{mg}$ FMAV has been also presented in (Duhamel et al. 2012). Note that very challeging mass and size of FMAVs can be reached nowadays with the progress of microelectronics. The on-board electronic circuit developed within the OVMI/EVA project weighs $100 \mathrm{mg}$ and is composed of a microprocessor, inertial and optic flow sensors. Piezoelectric actuators can reach $10 \mathrm{mg}$ and are used within FMAVs (Steltz \& Fearing 2007). Other low size and weight microelectronic devices exist on the market for such applications. The power supply remains very difficult to embark.

In the present work, the first strategy is adopted. For simulations, the physical data of a dipteran insect are used (Dudley 2002). The mass is of $200 \mathrm{mg}$ and the wingbeat frequency of $100 \mathrm{~Hz}$. The maximum amplitudes for flapping and rotation angles are taken respectively to $\phi_{0_{\max }}=60^{\circ}$ and $\psi_{0_{\max }}=90^{\circ}$. The wingspan and wings surface are assumed respectively to $2 L=3 \mathrm{~cm}$ and $2 S_{w}=1.14 \mathrm{~cm}^{2}$, allowing to generate a vertical ascendant movement using admissible flapping angles amplitudes.

Based on the defined numerical values, the admissible set for control torques $\Omega_{\bar{\tau}_{1}, \bar{\tau}_{3}}$ can be determined (2.17) and plotted (FIGURE 5).

$\Omega_{\bar{\tau}_{1}, \bar{\tau}_{3}}$ is approximated to the largest ellipse $E_{r}$ that fits inside $\Omega_{\bar{\tau}_{1}, \bar{\tau}_{3}}$ (FIGURE 5) for computation simplification reasons. The control torques $\bar{\tau}_{1}$ and $\bar{\tau}_{3}$ respect then an ellipsoidal admissible set defined by

$$
\left[\begin{array}{ll}
\bar{\tau}_{1} & \bar{\tau}_{3}
\end{array}\right] Q\left[\begin{array}{ll}
\bar{\tau}_{1} & \bar{\tau}_{3}
\end{array}\right]^{T} \leq 1
$$

where $Q$ is a diagonal definite positive matrix defining the ellipse's semi-axes denoted $a_{r}$ and $b_{r}$. Practically, if $\bar{\tau}_{1} \geq a_{r}(3.5), \bar{\tau}_{1}$ could be saturated to $a_{r}$, and $\bar{\tau}_{3}$ will be equal to zero. A privilege is given to the roll control in order to bring the FMAV to the horizontal plane. To avoid having a null yaw control torque and to give preference to the 
roll movement, $70 \%$ of $a_{r}$ is attributed to $N_{1}, \bar{\tau}_{3}$ will be calculated by (4.1) defining then a set $\Omega_{r}$ (FIGURE 5).

The control torque (3.5) is applied to the FMAV to be validated in simulation. Three sensors are embarked on the FMAV to mimic a part of the sensory system of an insect (halteres, legs sensilla and magnetic sense): three rate gyros, a tri-axis accelerometer and a tri-axis magnetometer whose models are given by $(2.3,2.5,2.4)$, respectively. The sensors measurements are not perfect, additive white gaussian noise is considered having a standard deviation of $\sigma_{G}=10^{-3} \mathrm{rad} / \mathrm{s}$ for the rate gyros, $\sigma_{A}=10^{-4} \mathrm{~m} / \mathrm{s}^{2}$ for the accelerometer and $\sigma_{M}=0.01$ mgauss for the magnetometer. Because of the low inertia of the FMAV, the developed control torque has also very small values of almost $10^{-5} N \cdot m$ (Figure 5). Since the control law is computed through a feedback of direct sensor measurements, the sensors should have a remarkable precision in order to guarantee a reasonable signal to noise ratio. Note that sensors with suitable precision or sensibility have already been developed (Cheinet et al. 2008). Even if the averaging of the angular velocity and attitude error reduce the noise influence, it can not be totally eliminated. The tuning parameters considered in simulations are given by $\left(N_{1}, N_{2}, N_{3}\right)=\left(0.7 a_{r}, 10^{-5}, \frac{b_{r}}{a_{r}} \sqrt{a_{r}^{2}-\bar{\tau}_{1}^{2}}\right)$ with $\left(a_{r}, b_{r}\right)=\left(1.859 \cdot 10^{-5}, 5.843 \cdot 10^{-5}\right)$, $\lambda=5 \cdot 10^{-6},\left(\rho_{1}, \rho_{2}, \rho_{3}\right)=\left(\frac{2.2 \cdot 10^{-3}}{a_{r}}, 15.5, \frac{5 \cdot 10^{-4} a_{r}}{b_{r} \sqrt{a_{r}^{2}-\bar{\tau}_{1}^{2}}}\right)$.

The evolution of the roll, pitch and yaw angles as well as the angular velocities measured by the rate gyros and the control torques are plotted in Figure 6 . Note that the body's angles are not used in the control torque, they are plotted only to show the convergence of the FMAV's orientation. The initial orientation is $\left(-40^{\circ},-25^{\circ}, 50^{\circ}\right)$ for the roll, pitch and yaw angles, respectively. The initial angular velocity is null. The sensor measurements are presented in FIGURE 7 and the wing angle amplitude envelops in FIgURE 8 . The stability is reached in a sufficiently fast time which makes the control law suitable for real-time implementation on a FMAV. Moreover, it presents comparable values to those observed in true insects (Dudley 2002). The rate gyros noise is detectable in FiguRE 6 . The accelerometer's noise is more detectable than that of the magnetometer. Their measurements converge to $s_{A}^{f}=[0,0,-1]^{T}$ and $s_{M}^{f}=\left[\frac{1}{2}, 0,-\frac{\sqrt{3}}{2}\right]$ at the equilibrium. Recall that the FMAV is stabilized in hovering mode. Therefore, the flapping angles converge to the value that generates an aerodynamic lift balancing its weight. The rotation angles converge to zero so that no translational movement is generated.

The robustness of the control law is tested with respect to external disturbances. These disturbances simulate wind or rain drops affecting the FMAV and creating a body torque of $\left(1.210^{-5}, 210^{-5}, 1.210^{-5}\right) \mathrm{N} \cdot \mathrm{m}$, applied at $t=1.5 \mathrm{~s}$ during 10 wingbeat periods. Noting that a rain drop weighs about $5 \cdot 10^{-6} N$ and the FMAV body's inertia is of $10^{-8} \mathrm{Kg} \cdot \mathrm{m}^{2}$, the disturbance applied has a great effect on the body's movement. It will destabilize the FMAV all over the disturbance taking it far away from the equilibrium. The control law acts later on to bring the FMAV back to stability. The evolution of the roll, pitch and yaw angles, angular velocities and control torques is plotted in Figure 9 zoomed to the disturbance zone in Figure 10. Note that the bound of the yaw torque depends on the value of the roll torque as explained previously. The reference sensor measurements are given in Figure 11 and the wing angle amplitudes in Figure 12. Notice that the flapping angle of the left wing is bounded during the disturbance at $\phi_{\max }=60^{\circ}$ avoiding the saturation of the actuator and emphasizing the development of a bounded control law. One should also note that an insect subject to such a high disturbance will lose all control of its position and orientation and regain it only when the disturbance is over. 

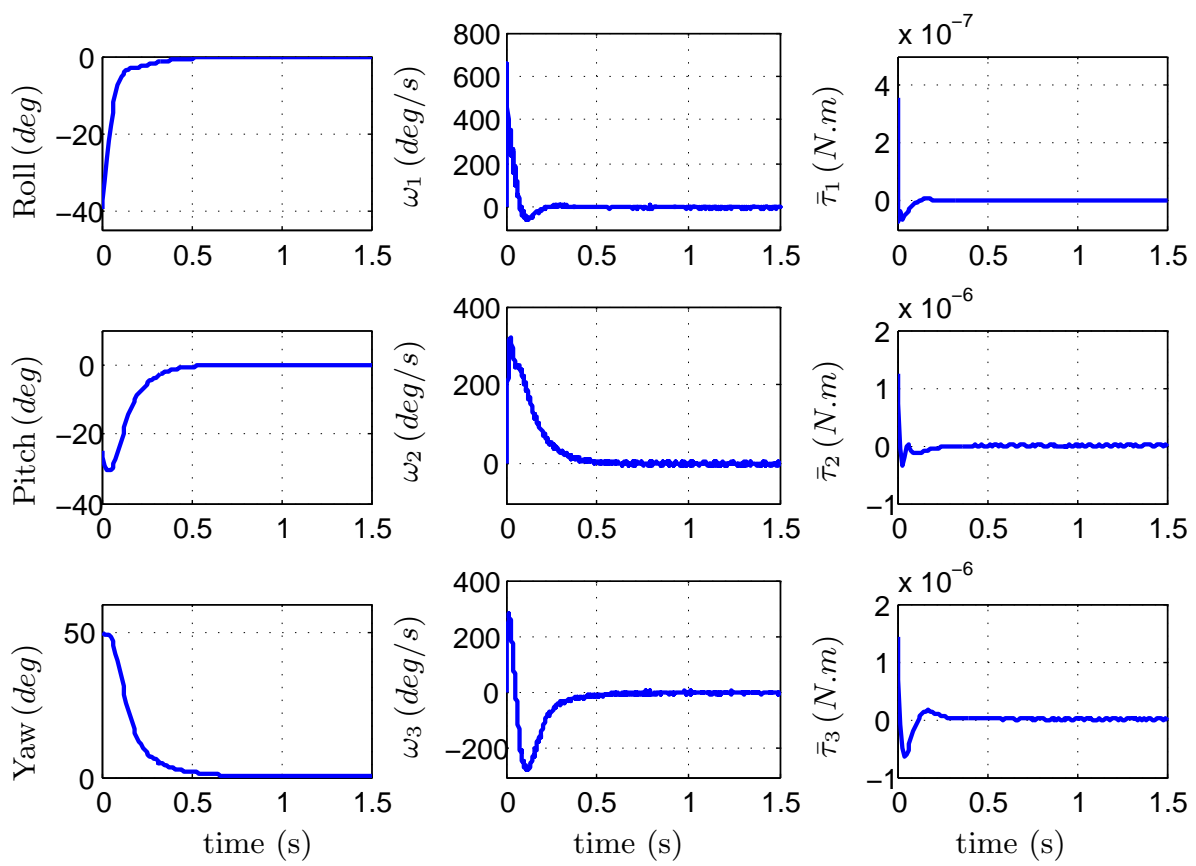

Figure 6. The attitude (left), angular velocity (middle) of the FMAV going from initial roll, pitch and yaw angles $\left(-40^{\circ},-25^{\circ}, 50^{\circ}\right)$ and null angular velocity and the control torques (right) applied to the FMAV.
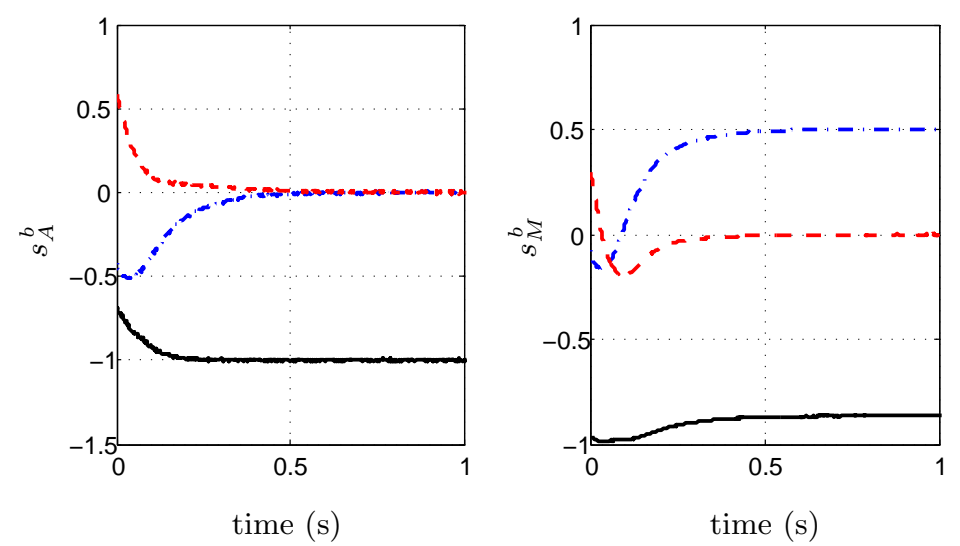

FIGURE 7. The reference sensor measurements: accelerometer (left) and magnetometer (right). The measurement along the roll axis is plotted with dot-dashed blue line, the pitch axis with dashed red line and yaw axis with continuous black line.

\section{Conclusions and future works}

Even if the gap between the flapping-wing animal flight and its technological reproduction is still very large, the robotic and control communities are exerting big effort to develop flapping micro aerial vehicles that mimic the nature's flight the most accurately possible.

The main contribution of this work is the development of a control law stabilizing the 

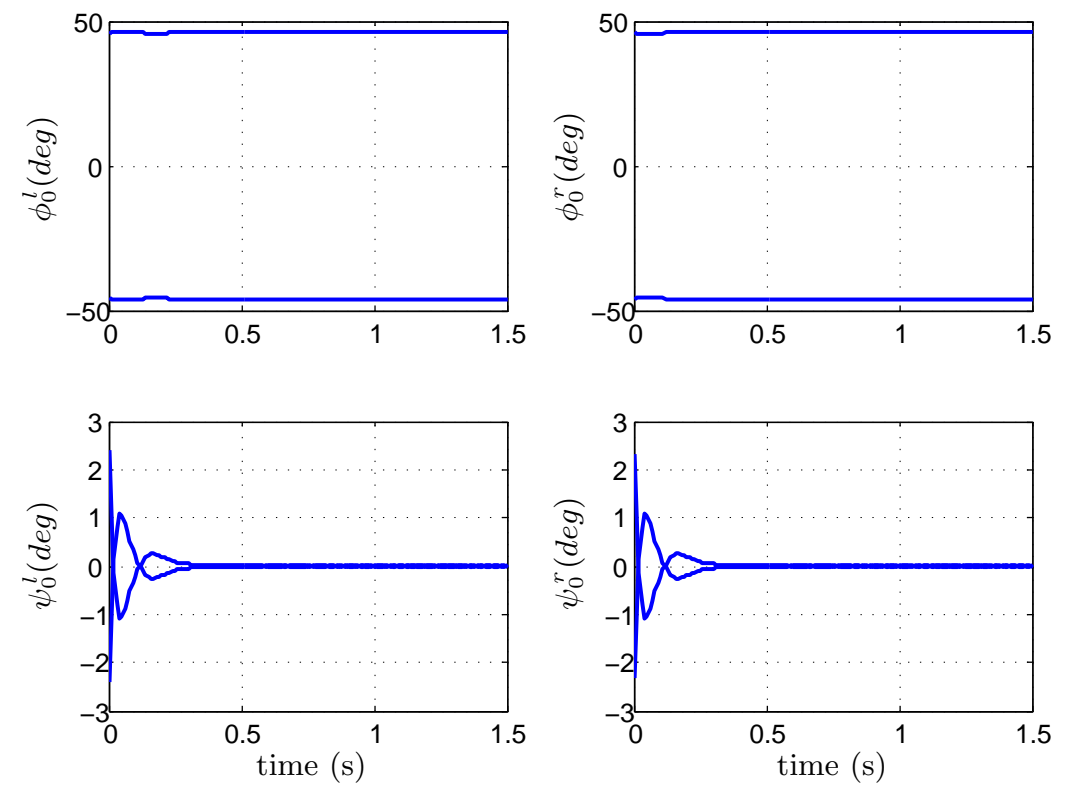

Figure 8. The envelops of the left and right wing angles.

FMAV's attitude. It is based directly on the measurements of some embedded sensors without the need of computing the orientation. This strategy reproduces the insects' one to stabilize their orientation based on their halteres, legs sensilla and magnetic sense. The control torque is bounded, allowing to take into account the amplitude bounds of the flapping wings in order to avoid the saturation of the actuators and ensure the stability of the body. Note that it allows different saturation bounds along the three axes. It is also not restricted to symmetric bodies and independent of the inertia matrix. Moreover, it is simple to compute and is adaptable for real time implementation. As shown in simulations, the control law is robust with respect to external disturbances. The boundedness of the control torque helps preserving the piezoelectric actuators not saturated and guarantee then their linear behavior even in hard conditions.

Future works will consider the development of bounded control force aiming to control the FMAV's trajectory based on sensor's measurements. In fact, insects can determine the sun direction using their ocelli. Moreover, the light polarization direction can be determined using the compound eyes. Based on polarized light compasses, for example, one can determine the direction of flight and couple it with the attitude control in order to ensure a movement in the three-dimensional space.

\section{REFERENCES}

Alexander, D.-E. 1986 Wind tunnel studies of turns by flying dragonflies. Journal of Experimental Biology 122, 81-98.

Alexander, D.-E. \& Vogel, S. 2004 Nature's flyers: Birds, insects and the biomechanics of flight. JHU Press.

Bullo, F. 2002 Averaging and vibrational control of mechanical systems. SIAM Journal on Control and Optimization 41 (2), 452-562.

Campolo, D., Barbera, G., Schenato, L., Pi, L., Deng, X. \& Guglielmelli, E. 2009 Attitude stabilization of a biologically inspired robotic housefly via dynamic multimodal attitude estimation. Advanced Robotics 23 (7-8), 955-977. 

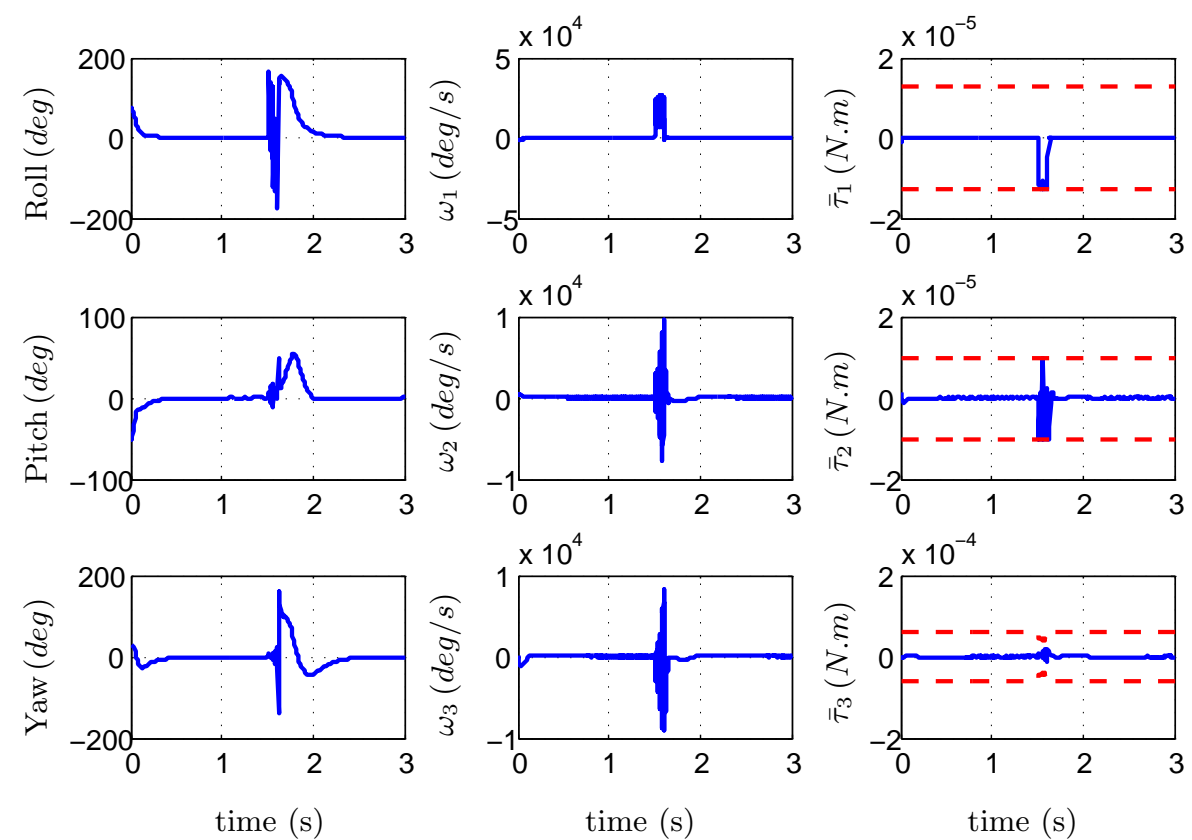

Figure 9. Robustness with respect to external disturbances: The attitude (left), angular velocity (middle) and the control torques (right) applied to the FMAV.
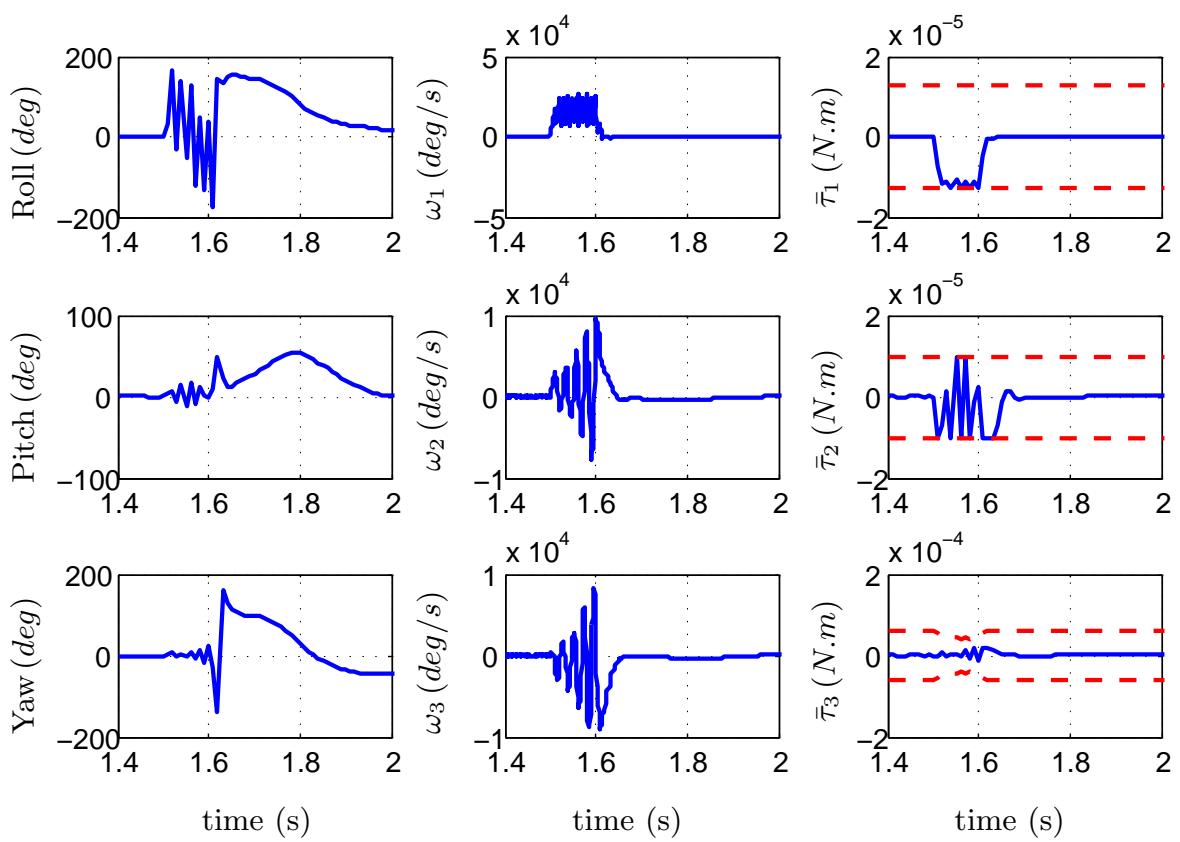

FiguRE 10. Robustness with respect to external disturbances: The attitude (left), angular velocity (middle) and the control torques (right) applied to the FMAV zoomed to the disturbance. 

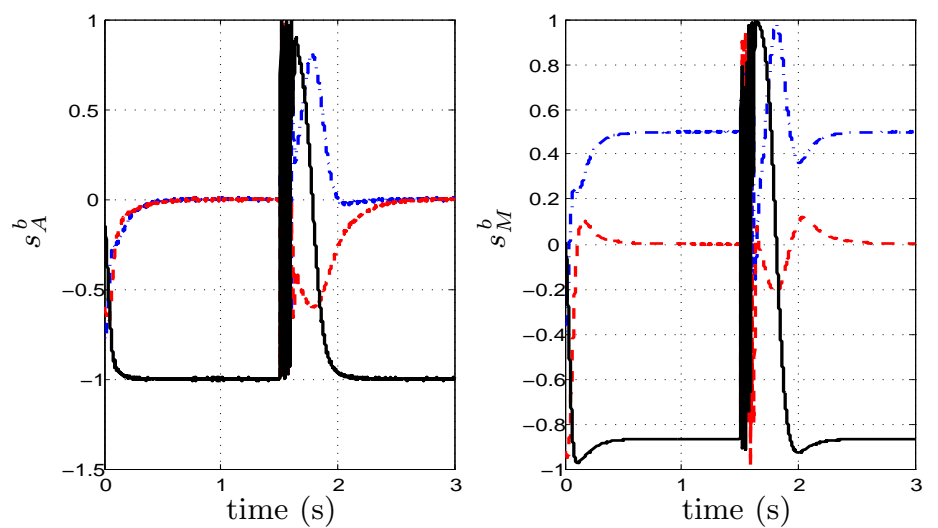

FiguRE 11. Robustness with respect to external disturbances: The reference sensor measurements: accelerometer (left) and magnetometer (right). The measurement along the roll axis is plotted with dot-dashed blue line, the pitch axis with dashed red line and yaw axis with continuous black line.
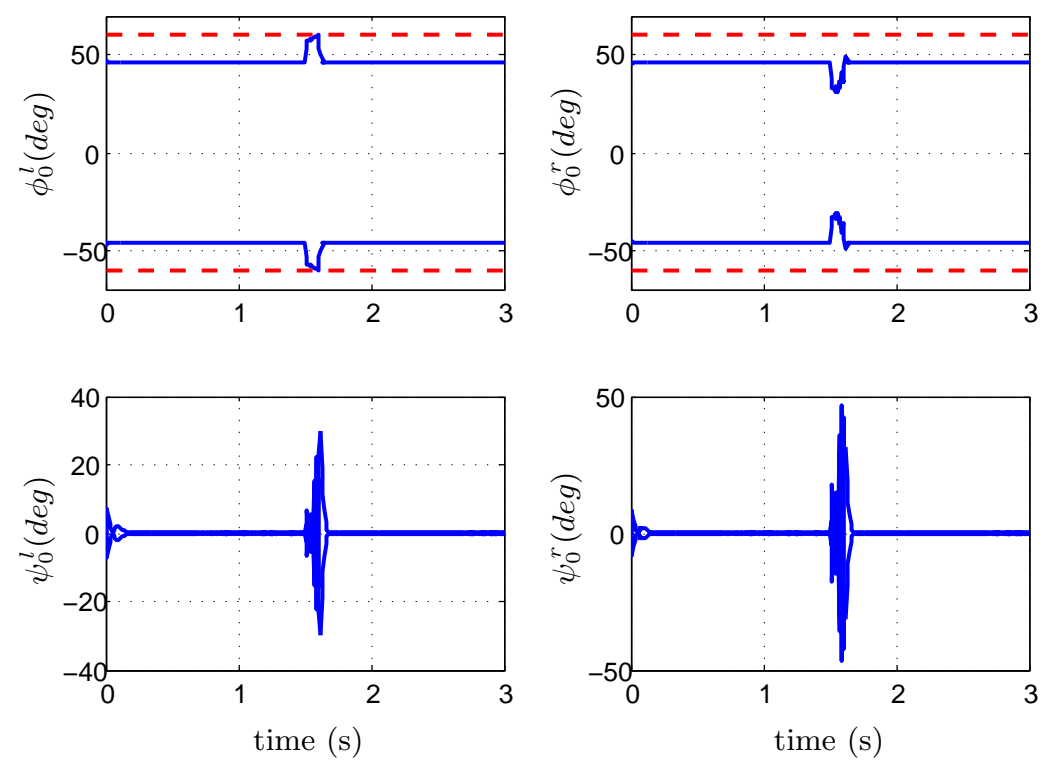

FiguRE 12. Robustness with respect to external disturbances: The envelops of the left and right wing angles. The bounds of the flapping angles are plotted with red dashed lines.

Campolo, D., Sitti, M. \& Fearing, R. S. 2003 Efficient charge recovery method for driving piezoelectric actuators with quasi-square waves. IEEE Trans. on Ultrasonics, Ferroelectrics and Frequency Control 50 (3), 237-244.

Chapman, R.F. 1998 The insects: Structure and function, $4^{\text {th }}$ edn. Cambridge University Press.

Cheinet, P., Canuel, B., Pareira Dos Santos, F., Gauguet, A., Leduc, F. \& LandraGIN, A. 2008 Measurement of the sensitivity function in time-domain atomic interferometer. IEEE Transactions on instrumentation and measurement 57 (6), 1141-1148.

Chung, S.-J. \& Dorothy, M. 2010 Neurobiologically inspired control of engineered flapping flight. Journal of guidance, control and dynamics 33 (2), 440-453. 
Couceiro, M.-S., Luz, J.-M., Figueiredo, C.-M. \& Fonseca Ferreira, N.-M. 2012 Modeling and control of biologically inspired flying robots. Robotica 30 (1), 107-121.

Deng, X., Schenato, L. \& Sastry, S. 2003 Model identification and attitude control for a micromechanical flying insect including thorax and sensor models. In Proceedings of the IEEE International Conference on Robotics and Automation, pp. 1152-1157. Taipei, Taiwan.

Deng, X., Schenato, L., Wu, W.-C. \& Sastry, S. 2006 a Flapping flight for biomimetic robotic insects: Part I- system modeling. IEEE Transactions on Robotics 22 (4), 776-788.

Deng, X., Schenato, L., Wu, W.-C. \& Sastry, S. $2006 b$ Flapping flight for biomimetic robotic insects: Part II- flight control design. IEEE Transactions on Robotics 22 (4), 789803.

Dickinson, M.H., Lehmann, F.-O. \& Sane, S.P. 1999 Wing rotation and the aerodynamic basis of insect flight. Science 284 (5422), 1954-1960.

Dudley, R. 2002 The biomechanics of insect flight: form, function, evolution. Princeton Univerity Press.

Duhamel, P.-E., Prez-Arancibia, N.-O., Barrows, G.-L. \& Wood, R.-J. 2012 Altitude feedback control of a flapping-wing microrobot using an on-board biologically inspired optical flow sensor. In Proceedings of the IEEE Intenational Conference on Robotics and Automation, pp. 4228-4235. St Paul, MN, USA.

Epstein, M., Waydo, S., Fuller, S.-B., Dickson, W., Straw, A., Dickinson, M.-H. \& Murray, R.-M. 2007 Biologically inspired feedback design for drosophila flight. In American Control Conference, pp. 3395-3401. New York, USA.

Finio, B.-M., Eum, B., Oland, C. \& Wood, R.-J. 2009 Asymmetric flapping for a robotic fly using a hybrid power-control actuator. In Proceedings of the International Conference on Intelligent Robots and Systems, pp. 2755-2762. St. Louis, MO, USA.

Finio, B.-M., Prez-Arancibia, N.-O. \& Wood, R.-J. 2011 System identification and linear time-invariant modeling of an insect-sized flapping-wing micro air vehicle. In Proceedings of the International Conference on Intelligent Robots and Systems, pp. 1107-1114. San Francisco, CA, USA.

Hedrick, T.L. \& DANiEL, T.L. 2006 Flight control in the hawkmoth Manduca sexta: the inverse problem of hovering. The journal of experimental Biology 209 (16), 3114-3130.

JANocha, H. \& StieBel, C. 1998 New approach to a switching amplifier for piezoelectric actuators. In Proceedings of the 6th International Conference on New Actuators, pp. 189192. Bremen, Germany.

Khalil, H.K. 2002 Nonlinear systems. Prentice Hall.

Kuhnen, K., Janocha, H., Thull, D. \& Kugi, A. 2006 A new drive concept for high-speed positioning of piezoelectric actuators. In Proceedings of the 10th International Conference on New Actuators, pp. 82-85. Bremen, Germany.

Lentink, D. \& Biewener, A.-A. 2010 Nature-inspired flight-beyond the leap. Bioinspiration and Biomimetics $\mathbf{5}$ (4).

Markley, F. L., Crassidis, J. L. \& Cheng, Y. 2006 Nonlinear attitude filtering methods. In AIAA Guidance, Navigation, and Control Conference.

Mountcastle, A.-M. \& Danie, T.-L. 2010 Vortexlet models of flapping flexible wings show tuning for force production and control. Bioinspiration and Biomimetics 5 (4).

Nachtigall, W. \& Wilson, D.-M. 1967 Neuro-muscular control of dipteran flight. Journal of Experimental Biology 47, 77-97.

Oppenheimer, M.-W., Doman, D.-B. \& Sightorsson, D.-O. 2009 Dynamics and control of a minimally actuated biomimetic vehicle: Part II-control. In proceedings of the AIAA guidance, navigation and control conference, p. 6161. Chicago, Illinois, USA.

Oppenheimer, M.-W., Doman, D.-B. \& Sightorsson, D.-O. 2010 Dynamics and control of a biomimetic vehicle using biased wingbeat forcing functions: Part I-aerodynamic model. In proceedings of the 48th AIAA aerospace sciences meeting, p. 1023. Orlando, Florida, USA.

Perez-Arancibia, N.-O., Ma, K.-Y., Galloway, K.-C., Greenberg, J.-D. \& Wood, R.J. 2011 First controlled vertical flight of a biologically inspired microrobot. Bioinspiration and biomimetics 6 (3), 036009.

Rakotomamonjy, T., Ouladsine, M. \& LeMoing, T. 2010 Longitudinal modelling and 
control of a flapping-wing micro aerial vehicle. Control Engineering Practice 18 (7), 679690 .

Renaudin, A., Zhang, V., Tabourier, P., Camart, J.C. \& Druon, C. 2004 Droplet manipulation using SAW actuation for integrated microfluidics. In $\mu T A S$, pp. 551-553. Malm, Sweden.

Rifai, H., Marchand, N. \& Poulin, G. 2008 Bounded control of a flapping wing micro drone in three dimensions. In Proceedings of the 2008 IEEE Int. Conf. on Robotics and Automation, pp. 164-169. USA.

SAne, S.P. 2003 Review The aerodynamics of insect flight. The journal of experimental Biology 206 (23), 4191-4208.

Schenato, L., Campolo, D. \& Sastry, S. 2003 Controllability issues in flapping flight for biomimetic micro aerial vehicles (MAVs). In Int. Conf. on Decision and Control. USA.

Schenato, L., Wu, W.-C. \& Sastry, S. 2004 Attitude control for a micromechanical flying insect via sensor output feedback. IEEE Transactions on Robotics and Automation 20 (1), 93-106.

Senda, K., Sawamoto, M., Kitamura, M., Obara, T. \& Kanazawa Univ., K. 2008 Effects of flexibly torsional wings in flapping-of-wings flight of butterfly. In World automation congress, pp. 1-6. Hawaii, USA.

Shyy, W., Aono, H., Chimakurthi, S.-K., Trizila, P., Kang, C.-K., Cesnik, C.E. \& LiU, H. 2010 Recent progress in flapping wing aerodynamics and aeroelasticity. Progress in Aerospace Sciences .

Sreetharan, P.-S. \& Wood, R.-J. 2011 Passive torque regulation in an underactuated flapping wing robotic insect. Autonomous robots 31 (2-3), 225-234.

Steltz, E. \& Fearing, R.-S. 2007 Dynamometer power output measurements of piezoelectric actuators. In Proceedings of the International Conference on Intelligent Robots and Systems, pp. 3980-3986. San Diego, California, USA.

Thomson, S.-L., Mattson, C.-A., Colton, M.-B., Harston, S.-P., Carlson, D.-C \& CutLER, M. 2009 Experiment-based optimization of flapping wing kinematics. In Proceedings of the $4^{\text {th }}$ AIAA Aerospace Sciences Meeting.

VelA, P. A. 2003 Averaging and control of nonlinear systems. PhD thesis, California Institute of Technology. 